\title{
Report from the Working Group on Diet and Cancer
}

\author{
Hans-Olov Adami, Lars Dragsted, Bent Enig, Jens Hansen, Jóhanna Haraldsdóttir, \\ Michael J. Hill, Lars Erik Holm, Ib Knudsen, Jens-Jørgen Larsen, Werner K. Lutz, \\ Merete Osler, Kim Overvad, Svend Sabroe, Tore Sanner, Michael Strube, \\ Thorkild I. A. Sørensen, Eivind B. Thorling.
}

\begin{abstract}
Michael Hill opened the session and gave an introduction by presenting a comparison between the 1985 and the 1991 European Cancer Prevention Organization (ECP) meetings on nutrition and cancer. In 1985 , specific recommendations regarding the intakes of fat, fibres, energy, fruit \& vegetables, alcohol and salt were given (see Michael Hill's article in this volume). The general trend was to weaken the statements regarding fat and fibres. The evidence for the beneficial effects of fruits and vegetables is strong. Obesity is to be avoided and that should be a public health priority. Food additives make an insignificant contribution to the cancer load. It was also pointed out that nutrition guidelines from the scientific community should in the future be directed to health promotion organizations to help them formulate a message to the public. Nutrition guidelines should be transmitted to professional communicators, who can then pase them on after integration with recommendations regarding not only cancer but all nutritionally related diseases.
\end{abstract}

The first session was concerned with

\section{The Occurrence of Carcinogens and Modifiers in the Diet}

Nutrition, Vitamins and Minerals in Denmark. The Danish population generally obtains a very large percentage of the energy from fat, $43 \%$, and there has been a steadily increasing trend over the last 30 years. The fat comes mainly from margarine and butter put on bread or used when preparing hot meals. A small increase in the consumption of fruits and vegetables has taken place since 1975. Relatively high levels of vitamin A are contained in the Danish diet, whereas levels of protective antioxidant factors like selenium, ascorbates, tocopherols and $\beta$-carotene are moderate to low. Reduction in the fat intake would be likely to result in an increase in the levels of water soluble vitamins.

Oxidative damage to the genome from natural cellular processes my exceed 10,000 adducts cell/day and may be an important contributor to the cancer load. It was proposed that this line of research should be pursued since it offered promising outlooks for more comprehensive theories that might explain a number of observations in the links between diet and humen cancer.

Fat. The total fat intake varies with a number of social variables and life style factors. Body mass index is a poor parameter for the fat content of the body and should be replaced by body impedance measures of the fat and lean body mass in future epidemiological studies. Seven types of cancer may be related to fat consumption in one way or another, namely, cancers of the breast, colon, rectum, endometrium, ovary, prostate, and gall bladder. Whether the relationship is causal, and if so, whether it depends on the high contribution that fat (as total fat, or as saturated, monounsaturated or polyunsaturated fatty acids) makes to the total caloric content of the diet is far from clear and may well vary from one type of fat-related cancer to another.

Alcoholic beverages. In the period from 1955 to 1990 , the yearly consumption for adult individuals in Denmark has increased from around 4l to 11-12 I of pure ethanol. This corresponds to about 2-3 drinks per adult per day. Concomitantly an increase in acute diseases related to heavy drinking has taken place. IARC has recently classified alcoholic beverages as carcinogenic to humans. The most direct avenue for reducing the cancer burden associated with drinking is to reduce the level of intake of alcoholic beverages. Because a substantial proportion of alcohol-induced oral, oesophagus, laryngeal, and hepatic cancers (the tumours most strongly related to alcohol) results from heavy drinking, reduction to moderate levels should considerably lower the risk of these cancers. However, evidence also exists that daily alcohol consumption even at moderate levels increases cancer risks.

Naturally occurring carcinogens in food. It was generally assumed that naturally occurring carcinogens might not represent a major human cancer risk. It should be stressed, however, that just a minor part of naturally occurring substances in our food has been adequately tested for mutagenicity/carcinogenicity.

Aflatoxins are classified by IARC as a human carcinogen and can induce liver tumours. They are produced by molds under hot and humid conditions, and can be detected in e.g. maize, peanuts and dried fruit. Aflatoxin $M_{1}$ may be found in dairy products. Aflatoxin contamination of human foods is not accepted in 
Denmark, so the practical limits of detection serve as acceptance limits. Generally the aflatoxin exposures to the Danish population are believed to be decreasing. They are estimated to cause from 0.5 to 5 cancer cases a year in Denmark.

Ochratoxin is considered carcinogenic. A tolerable daily intake (TDI) of $5 \mathrm{ng} / \mathrm{kg}$ bw has recently been determined by a Scandinavian working group. The regulation of food products for sale in Denmark is under consideration. The potential role of ochratoxin in human cancer needs further study.

Cooked food contains a variety of mutagenic heterocyclic amines. All the mutagenic heterocyclic amines so far tested were carcinogenic in rodents. Evidence exists of high cancer-provoking potency in primates. Suggestive evidence indicates a role of fried foods in colo-rectal cancers in humans, but studies on the specific role of heterocyclic amines are not available. Human studies on the specific role of cooked food mutagens are warranted, because a major problem in estimating their contributions is their correlation with animal fat consumption. Total avoidance of exposure to heterocyclic amines is impossible, but reducing the exposure level appears advisable. This can be done by reducing the frying temperature and time and to cook by other means than frying.

Fibre. A clear protective effect of fibres in human or in animal studies has not been consistently demonstrated. This may in part have been caused by deficient methods for fibre definition and determination. Much better characterization of physico-chemical properties of the fibre studies has to take place in order to obtain relevant information. Also, the effect of the sum of components that serve as pabulum for bacteria in the large bowel, including undegraded "resistant" starch must be studied more carefully.

Food additives. The actual human exposures to food additives in Denmark are below the acceptable daily intake (ADI). It is highly unlikely that food additives in Denmark today represent any significant cancer risk. The wisdom of the use of "cosmetic" additives was questioned.

Fruit and vegetables. An increased intake of vegetables and fruits are consistently associated with reduced risk of a number of different types of cancer in epidemiological studies. A number of substances with tumour-inhibiting properties have been identified in rodent studies, including vitamins like ascorbic acid, tocopherols, and carotenoids as well as many other compounds like isothiocyanates, dithiothiones, flavones, indoles, polyphenols, terpenes, and allylsulphides. In human studies, some vitamins have been implicated as tumour protective factors, but it is not fully known to what extent they are merely markers for other compounds. The variation in content of these compounds in various vegetables and fruits grown under different conditions are not well known, and exact human exposures are difficult to evaluate. Many of the cancer-protective agents are in fact antioxidants or stimulate natural antioxidation mechanisms, and it was concluded that the potential impact of such compounds on human cancer rates is particularly important to study.

\section{Risk Assessment}

Different methods have been used in assessing the importance of diet in human carcinogenesis, both in vitro, in vivo and epidemiological methods.

Fow carcinogens have so far been identified in the diet, some genotoxic and some non-genotoxic. Most of these carcinogens are not nutrients but components following the diet, e.g. pesticide (synthetic and "natural") residues or mycotoxins formed during growth and storage of foods. Finally a fow food additives have been discussed. All of these substances appear for the moment to be of minor importance in human carcinogenesis. The only future use of in vitro tests in diet-cancer risk assessment may be in preliminary evaluation of new substances to be introduced on the market.

Most dietary components considered appear to be modifiers of carcinogenesis or non-genotoxic carcinogens. These are best studied in animal experiments, but most of the experiments conducted so far have conaidered only variations in single components of the diet. In the human diet a mixture of nutrients is eaten with a concomitant large variation in many nutrients. This points to mechanistic studies in animals and risk assessment based on epidemiological studies.

The combined effect of known dietary and environmental exposures on the effect on human icancer incidence was calculated. The calculations were based on the best estimates from animal experiments and additive effects were assumed. No attention was paid to possible anticarcinogens in the diet. The conclusion was that even the most conservative estimation of the number of cases that could be explained this way was very low compared to the actually observed number of cases. This demonstrates a major deficit in our knowledge about cancer causes and stresses the importance of new approaches in the field of human carcinogenesis, e.g. the possible role for the metabolically generated oxygen-derived radicals.

There are different design options in epidemiology, for example case-referent studies, cohort studies and intervention trials.

In case-referent studies information on past diet is obtained from cases with the disease of interest and from a relevant reference group. Recalling past diet is very difficult and some studies indicate that the major predictor of the answers is present diet. It may therefore 
be possible to get relatively good information from people with stable diets, but in most studies considerable misclassification must be expected. Study power is therefore reduced and bias introduced in determining risk estimates. Further, the cases may have changed their diet habits due to the developing disease, and further bias may have been introduced. For these reasons case-referent studies will probably be of minor importance in future diet-cancer risk assessment.

Cohort studies have been suggested to overcome some of the difficulties in case-referent studies, and several studies are being planned all over Europe and some are already in progress. Most scientists agree that the cohort studies may prove of great scientific value especially when dietary information is combined with studies on collected biological material. It must, however, be assured that the collected information has the potential of being relevant after $10-15$ years, the problems of collinearity must be considered, and it must be assured that now information can be abstracted. One of the major problems in these studies will be to ensure that the study power is sufficient. The cohorts must be large, the populations must have sufficiently heterogeneous dietary habits, and all efforts must be taken to evaluate and/or reduce measurement error. Study power may be increased if collaborative studies and data analyses are undertaken, e.g. through European collaboration including use of standardized, calibrated methods. Focus in the cohort studies should be both on dietary patterns, foods, and nutrients with due attention to other social and life style factors.

The problems concerning intervention trials were reviewed. Some studies are in progress especially in populations at high risk of developing cancer either due to exposure to known carcinogens (smokers, asbestos workers, tin miners) or due to diseases known to increase cancer risk (colonic adenomatous polyps, leucoplakia, cervical dysplasia, asbestosis). It seems possible to conduct dietary intervention trials in these groups, especially when the intervention can be taken as supplements, which is the case in many studies on antioxidants. Pilot studies are in progress to evaluate the possibility of conducting dietary intervention trials among ostensibly healthy individuals - especially trials aiming at reducing total fat intake. Compliance in the intervention group seems promising, but unfortunately the reference group is receiving advise from other sources to reduce dietary fat intake, and it is difficult to predict the actual dietary differences between the compared groups in the long run. Further, these dietary intervention trials are extremely expensive and long lasting.

\section{Risk Management and Prevention}

The classification of dietary carcinogens, the Danish government's strategy for regulation of this area, and the international coordination of the regulations were presented. The three most important steps to reduce dietary cancer risk are to establish

- more precise estimates of etiologic fractions posed by different dietary habits

- more precise knowledge about individual dietary

factors, e.g. individual fatty acids and "fibre" components

- more knowledge about the relative importance of the different stages in cancer development.

It was emphasized that in the future we should watch out for interactions between a dietary regimen as a whole and the carcinogenic/anticarcinogenic potential of individual food items, and interaction between prevention of cancer and other health endpoints. Finally, it was stressed that a zero-risk society is an impossibility that neither science nor regulatory bodies can create.

The fundamental principles for giving food recommendations to the public are: that they must be consistent, credible, relevant and have practical application. However, at present there is hardly any information on how the Danish public perceives the food recommendations as to their credibility, relevance etc. Today the central issue is not primarily a lack of consensus among experts. The issue is rather how to deal with the fact that government agencies and voluntary heaith organizations are not the only actors on the food-advice scene. The average consumer cannot distinguish the different actors' agendas and potential biases. In the process of formulating food recommendations, a number of contradictions arise. These can only be solved in a satisfactory way if recommendations are planned on a long-term basis.

The complicated issue of multifactorial causality in relation to the development of chronic disease, especially cancer, was discussed. The use of the expression 'causes', in relation to the occurrence of cancer in man should be used with the greatest care, and preferentially avoided. Risk factors (positive or negative) would be a more appropriate description of the observed correlations. Use of modern statistical methods should be used to approach specificity in a causal network.

The possible problems with recommending and eating a diet meeting the recommendations to prevent cancer were pointed out. If this diet is such that it is perceived as non-palatable or as a sacrifice, then it should only be recommended to high-risk groups. However, the experience from the Stockholm Cancer Prevention Program is that the prudent cancer preventive diet can be made both palatable and pleasant. Given these conditions there would be no reason not to recommend such diet to the general population.

In the discussion several issues were stressed. Dietary recommendations should be more specific and give practical advice on what types of foodstuffs to choose and how to prepare them. We can only recommend one diet that has to encompass not only prevention of cancer 
but also other diet-related diseases. The dietary recommendations given by medical experts must be translated by health information professionals and channelled through appropriate organizations in order to get optimal effect and credibility. Scientists are notoriously poor advisers to the general public.

The main conclusions of the session were that the data presented at this workshop had shown that there is now a solid basis for recommendations to eat more fruits and vegetables to reduce cancer risks. We are now beginning to understand the mechanismo for their beneficial effects. Further, the importance of avoiding excessive caloric intake to reduce cancer risks was stressed. Finally, the group was of the opinion that food additives and contaminants only play a minor role in human carcinogenesis of today. 